\title{
An Oblivious Watermarking Scheme Based on Coutourlet Transform and Quantization Index Modulus Modulation
}

\author{
Dongfang Chen ${ }^{1}$, Bing Zhou \\ College of Computer Science and Technology, Wuhan University of Science and Technology,Wuhan,Hubei \\ 430065, P.R. China
}

\begin{abstract}
This paper presents an oblivious watermarking scheme based on contourlet transform and quantization index modulus modulation (QIMM).The scheme has the following features. First, the proposed scheme makes better use of multidirectional characteristic of contourlet transform and embed the watermark into the 4 directional high pass sub bands. Second, the scheme avoids the problem of selection of wavelet filter banks since approximately the same results are obtained with different contourlet filter banks in watermark embedding and extracting stage. Third, unlike the conventional watermarking schemes, watermark error correction is integrated to enhance the quality and normalized cross correlation (NC) value of the extracted watermark. Fourth, through the introduction of the error correction, the proposed scheme tries to view $\mathrm{NC}$ as a standard to judge the watermarking algorithm. As a comparison, experiments are conducted to demonstrate that our scheme is more effective than the former one based on lifting scheme wavelet transform.
\end{abstract}

Keywords: Contourlet transform, Encryption, QIMM, Error Correction, Image Watermarking

\section{Introduction}

With the development of digitization and World Wide Web, digital information becomes available to a large number of people. How to effectively protect information security and authors' ownership has been attracting more and more attention. Digital watermarking technology is developing fast due to its possibility to solve the problem.

The digital watermarking technology refers to revising the media and hiding data or information which is often imperceptible visually and can be recovered even in the media that have been compressed or edited. It is used to identify the owner and discourage unauthorized copying and distribution. The embedded watermarks should be transparent and robust. Transparency refers to that the watermark should be perceptually invisible. Robustness is the ability to resist signal processing or geometric attacks.

1 Corresponding author.

Email address: df_chen@163.com
Existing digital watermarking schemes can be classified into private and public (also called blind) depending on the requirement of the original image during the extracting process; most existing methods are private and typically they subtract the original image from the watermarked one. These methods are often robust owing to its private characteristics. In contrast, blind watermarking techniques are not that robust and therefore more suitable for applications requiring lower security such as authorized copying distribution in electronic commerce.

The digital image watermarking techniques are grouped in two classes: the spatial domain techniques which focus on rapid pixel-wise operations but are often prone to geometric attack, noise, and JPEG compression and the frequency domain techniques which embed the watermark in the domain of an invertible transform. The frequency domain techniques are of more concern since they are more robust than spatial domain methods. The main advantages of frequency watermarking schemes are: 1) the embedded 
watermark signal energy can be distributed to all pixels to guarantee the transparency of watermarks; 2) some characteristic of the visual system can be more easily integrated into the process of encoding watermark 3) the methods and compatible with international data compression standard. The most commonly used techniques are DCT (discrete cosine transform)[4], DFT(discrete fourier transform)[5][6], DWT(discrete wavelet transform)[2][3] and LWT (lifting scheme wavelet transform)[13].

In 2005 Minh N D and Martin Vetterli proposed the contourlet transform. Compared with the limitations of the conventional multiresolution image representations, the contourlet transform can efficiently capture the intrinsic geometrical structure of image which is key in visual information and can be utilized to complement the current watermarking techniques. Several existing schemes such as in [8][9][10][12] have been proposed in contourlet domain. In addition to direct work on watermarking, [11] proposed a method for extracting the significant features of an image from the contourlet coefficients to generate authentication signatures.

This paper presents an oblivious watermarking scheme in contourlet domain. Superiority of the proposed scheme is observed over the scheme proposed in [13] which is based on lifting scheme wavelet transform (LWT) and watermark scrambling encryption.

The paper is structured as follows. In section 0 , related techniques are introduced. In section 0 , watermarking embedding and extraction related issues are reported. Experimental results are included in section 0 . Finally, section 0 draws some concluding remarks and includes proposals of future work.

\section{Background}

\subsection{Discrete Contourlet Transform}

Wavelets in 2 dimensions are good at isolating the discontinuities at edge points. However, they can not "see" the smoothness along the contours. In addition, separable wavelets can capture only limited directional information which is an important and unique feature of multidimensional signals. Contourlet transform was proposed as a new image representation since its expansion has such features as multiresolution, directionality and anisotropy.

Contourlet transform which is also called pyramidal directional filter bank (PDFB) combines laplacian pyramid (LP) to capture the point discontinuities with a directional filter bank (DFB) to link these point discontinuities into linear structures. Figure 1 is the pyramidal directional filter bank of contourlet transform. For one level contourlet transform, the host image $a_{L}$ is decomposed into a sub band image $b_{L+1}$ and a lowpass image $a_{L+1}$.The band pass image $b_{L+1}$ is further processed by DFB. Figure 2 is the DFB which partition the frequency into $2^{\mathrm{k}}$ sub bands.

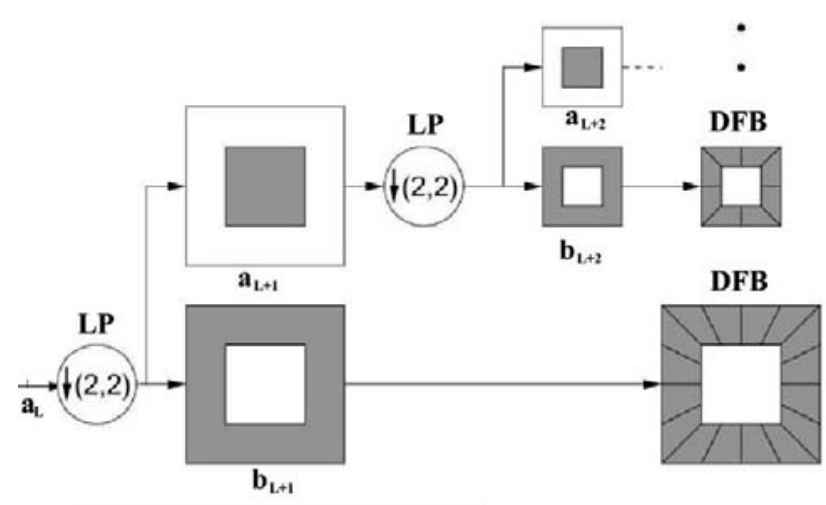

Figure 1.Pyramidal directional filter bank of contourlet

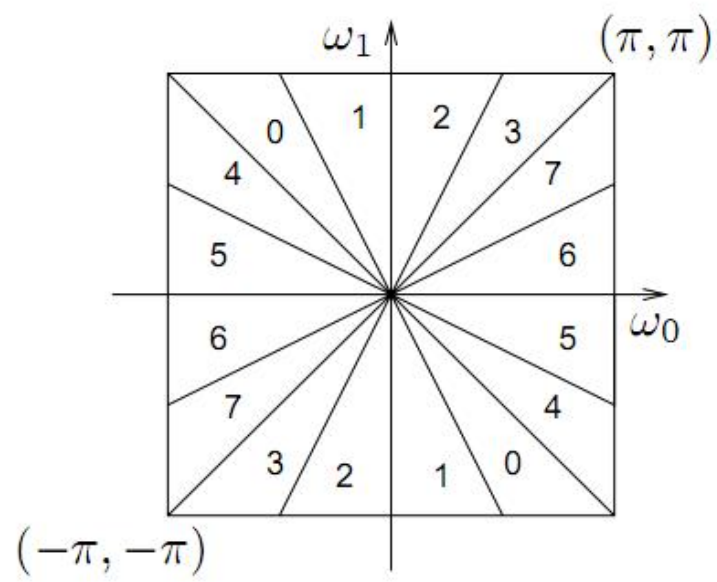

Figure 2.Frequency partitioning $\left(\mathrm{k}=3,2^{\mathrm{k}}=8\right.$ wedge shaped frequency sub bands)

Contourlet transform can be illustrated by decomposing the lena image of size $512 \times 512$ into 3 levels as shown in Figure 3. The top most band shown in Figure 3 is the low pass image. At each successive level the number of directional sub bands is 2,3,3. Last 4 directional sub bands are selected for watermark embedding.

\subsection{Watermark scrambling encryption}

To improve security, watermark must be encrypted. If someone has extracted the watermark, without secret key he can't decrypt the watermark and have no access to the true information. In addition scrambling encryption eliminates the spatial 
relevance among pixels.

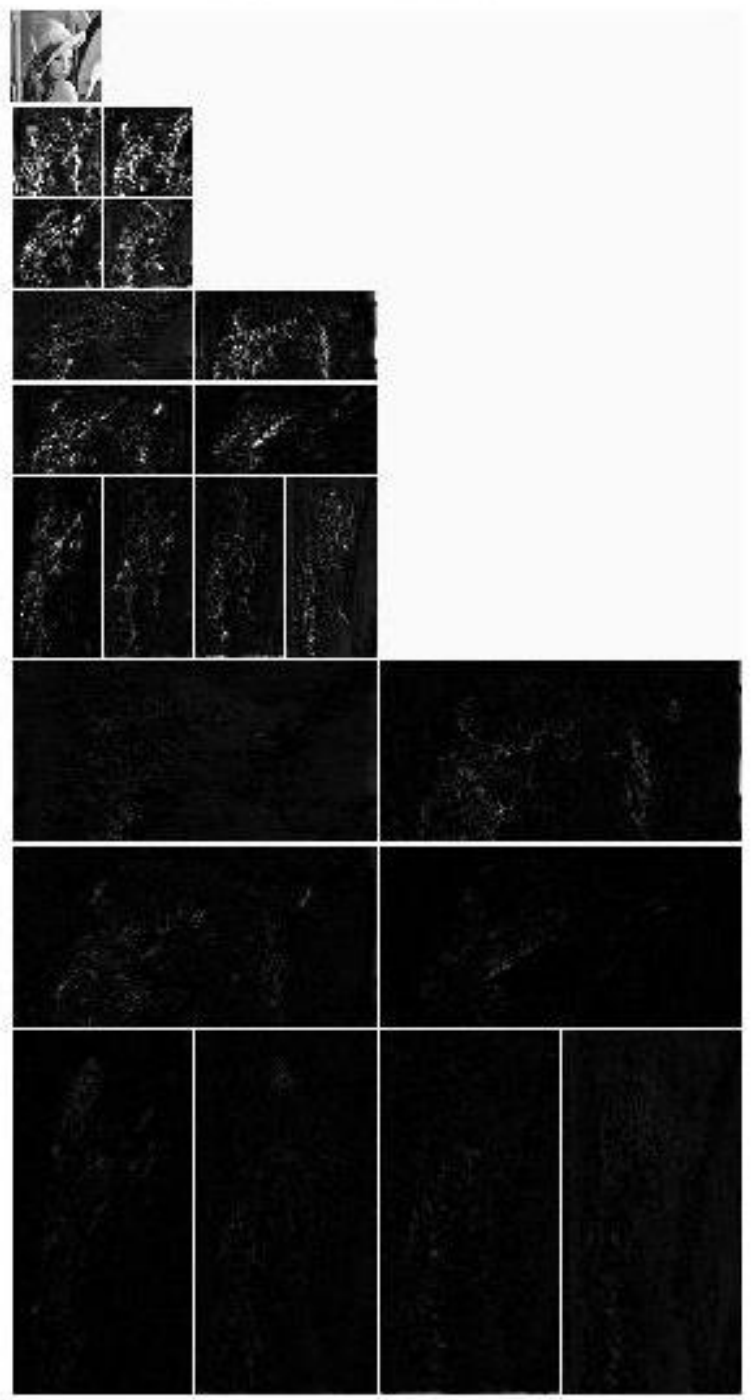

Figure 3.Contourlet decomposition of Lena

The scrambling encryption method is based on the cat chaotic mapping, a discrete chaotic modal proposed by the Arnold and Avez, which is also called Arnold transform. The Arnold transform is defined as follows:

$$
\left(\begin{array}{l}
x_{n+1} \\
y_{n+1}
\end{array}\right)=\left(\begin{array}{ll}
1 & 1 \\
1 & 2
\end{array}\right)\left(\begin{array}{l}
x_{n} \\
y_{n}
\end{array}\right)(\bmod N)
$$

If we apply Arnold transform to the watermark, the watermark layout will be changed since the pixel coordinates have been changed. Arnold transform is widely used in digital watermarking since it's simple and cyclical. When we repeatedly apply Arnold transform, at some point the watermark will be recovered. However, it's time-consuming to restore the watermark through its cycle because its cycle is proportional to the size of the matrix. In practice, we take the count of transform as secret key and decrypt the image with the inverse transform. Figure 4 is the original watermark and the encrypted watermark after 20 times Arnold transform.

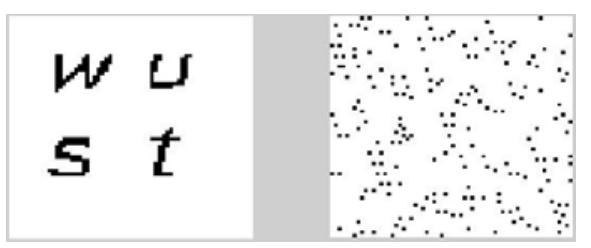

Figure 4 .The original watermark(left) and the encrypted watermark(right)

\subsection{Quantization Index Modulus Modulation (QIMM)}

There are three common quantization methods: (1) QIM (Quantization Index Modulation)[7]; (2) DM (Dither Modulation); (3) QIMM (Quantization Index Modulus Modulation). In this paper, we use the QIMM method which we shall describe in details in this section. The proposed QIMM approach selects some of contourlet coefficients as the original host signal. The index of each quantized coefficients is modulated for embedding one bit of information. The embedding and extraction processes are described as follows.

In the embedding process, suppose the host signal is $X=\left\{x_{1}, x_{2}, \ldots, x_{n}\right\}$ and the watermark bit sequence is $W=\left\{w_{1}, w_{2}, \ldots, w_{n}\right\}$, where $w_{i} \in\{0,1\}$. To embed one watermark bit, the embedding algorithm consists of the following steps:

(1) Let $\mathrm{Q}$ be the quantization step size , figure out the following value:

$$
\begin{aligned}
& v_{1}=\bmod \left(x_{i}, Q\right) \\
& v_{2}=x_{i}-v_{1}
\end{aligned}
$$

(2) Modify the host signal using the following formula:

a) If $w_{i}=0$,

$$
v_{1}^{\prime}=\left\{\begin{array}{cl}
\frac{Q}{8} & v_{1}<\frac{3 Q}{8} \\
\frac{5 Q}{8} & \frac{3 Q}{8} \leq v_{1}<\frac{7 Q}{8} \\
\frac{9 Q}{8} & \frac{7 Q}{8} \leq v_{1}<Q
\end{array}\right.
$$

b) If $w_{i}=1$,

$$
v_{1}^{\prime}=\left\{\begin{array}{cc}
-\frac{Q}{8} & v_{1}<\frac{Q}{8} \\
\frac{3 Q}{8} & \frac{Q}{8} \leq v_{1}<\frac{5 Q}{8} \\
\frac{7 Q}{8} & \frac{5 Q}{8} \leq v_{1}<Q
\end{array}\right.
$$


At last, the modified host signal value is:

$$
x_{i}^{\prime}=v_{2}+v_{1}^{\prime}
$$

After receiving the attacked watermarked signal $X^{\prime}=\left\{x_{1}{ }^{\prime}, x_{2}{ }^{\prime}, \ldots, X_{n}{ }^{\prime}\right\}$, the watermark bits $w_{i}{ }^{\prime}$ are extracted as follows.

(1) For $x_{i}^{\prime}$, figure out $v_{1}$ and $v_{2}$ with the formula (2) and (3) where the quantization step size is the same as that in watermark embedding stage.

(2) Determining the watermarking bit value according to following formula:

$$
W_{i}^{\prime}= \begin{cases}0 & 0 \leq v_{1}<\frac{Q}{4}, \text { or } \frac{Q}{2} \leq v_{1}<\frac{3 Q}{4} \\ 1 \quad & \frac{Q}{4} \leq v_{1}<\frac{Q}{2} \text {, or } \frac{3 Q}{4} \leq v_{1}<Q\end{cases}
$$

\section{The Proposed Scheme}

\subsection{Frequency Coefficients Selection}

In this paper, contourlet transform is applied to the image. With 3 level LP decomposition and DFB directional decomposition, the image is decomposed into a lowpass sub image and a number of directional sub bands. The watermark should not be embedded in the lowpass sub image, since the sub image is an approximation of the original image, whose distortion can be easily perceived. Also according to the experiments conducted by physiologists, the human eye has the following characteristics: 1) the human eye has low sensitivity to the additional noise in high and bright regions of images; 2) the human eyes have relatively low sensitivity to the image texture regions compared with the smooth regions; 3) the human eye has very low sensitivity to high-frequency information of images. Considering the watermark embedded in the first 2 LP level sub bands is prone to JPEG attack, the watermark should be embedded in the last level directional sub bands so that the watermark is imperceptible and can resist JPEG compression and common signal processing such as sharpening and blurring.

\subsection{The Watermark Embedding}

Steps of embedding algorithms are as follows:

(1) The contourlet transform is applied to the host image of size $N_{1} \times N_{1}$. A three $\left(l_{1}, l_{2}, l_{3}\right)$ level pyramidal structure is selected for LP decomposition. At each level $l_{k}$, there are $2^{l_{k}}$ directional sub bands, where $k=1,2,3$.Last level directional sub image $c_{3,0}^{(2)}, c_{3,1}^{(2)}, c_{3,2}^{(2)}, c_{3,3}^{(2)}$ are selected for watermark embedding whose size is $\frac{N_{1}}{2^{3}} \times \frac{N_{1}}{2^{3}}$.

(2) K-time Arnold transform is applied to the watermark $W$ of size $M_{1} \times M_{1}$. The time $\mathrm{K}$ is recognized as secret key to decrypt the watermark. The encrypted watermark $W^{\prime}$ is split equally into 4 parts $W_{0}^{\prime}, W_{1}^{\prime}, W_{2}^{\prime}, W_{3}^{\prime}$, since we will embed them into the 4 directional sub images in the last LP level.

(3) $W_{i}^{\prime}$ will be embedded into $c_{3, i}^{(2)}$ where $\mathrm{i}=0,1,2,3$. The directional sub band image is divided into blocks of size $M_{2} \times M_{2}$, where $M_{2}=\frac{N_{1}}{2^{2} \times M_{1}}$. There are $\frac{M_{1}}{2} \times \frac{M_{1}}{2}$ blocks in total. The bits of $W_{i}^{\prime}$ will be embedded into the left up point of blocks using the QIMM method proposed in section 0. The quantization value is used later in watermark extraction.

(4) The inverse contourlet transform is applied to the modified sub bands and the final watermarked image is obtained.

Figure 5 is the involved embedding steps.

\subsection{The Watermark Extraction}

In the extraction stage, the required parameters are quantization step size, watermark size and Arnold transform time. Steps of watermark extraction algorithm are as follows.

(1) The contourlet transform is applied to the watermarked image of size $N_{1} \times N_{1}$ and we get 4 last level directional sub bands $c_{3,0}^{(2)}, c_{3,1}^{(2)}, c_{3,2}^{(2)}, c_{3,3}^{(2)}$ of size $\frac{N_{1}}{2^{3}} \times \frac{N_{1}}{2^{3}}$.

(2) Get the contourlet coefficients that contain watermark bits information according to the position-to-position mapping in the embedding process. Extract the watermark using the QIMM method in section 0. With this process, we get 4 watermark images since there are 4 directional sub bands.

(3) Merge the 4 images obtained from the above procedure and the encrypted watermark is extracted from the image. The inverse Arnold transform is applied to the encrypted watermark and the final watermark is obtained.

\subsection{Watermark Error Correction}

The watermarked image may be distorted by intentional or unintentional manipulations; 
consequently the extracted watermark undergoes information lost or error which results in the ambiguity in the authentication stage. So a technique should be involved to correct the distorted watermark to improve the accuracy.

For watermark image, the watermark bits may be separated into important bits and unimportant bits by the human visual system. Normally the distortion in unimportant bits makes the important information undistinguishable and thus must be corrected.

Since we use a text image as watermark, the expected distortion is gaussian noise which affects the recognition of important bits or text. The proposed algorithm is as follows:

(1) Scan the extracted watermark, and find the connected domain using the concept of 8-connected domain.

(2) Check whether the domain width and height are both less than a given threshold or not, If it's true, the connected domain is discarded and set to 1(white color); else the connected domain is preserved.

After the error correction, much random noise is discarded, which enhance the watermark quality and improve the NC value.

Figure 6 is the extraction steps.

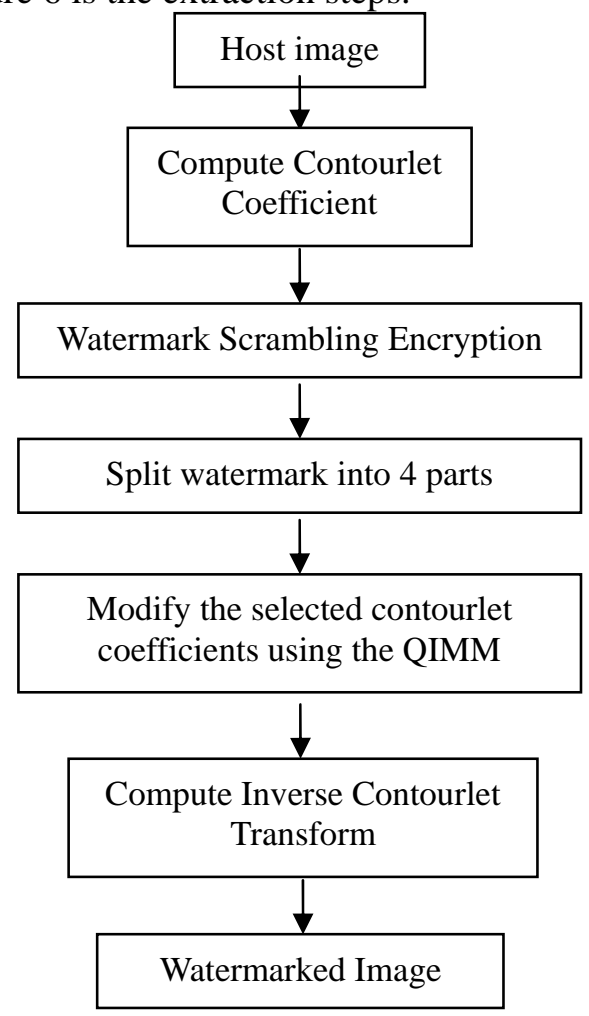

Figure 5.Steps of watermark embedding
Lena image of size $512 \times 512$ is considered as host image. The watermark image is a binary logo having the letters 'wust' of size $64 \times 64$.Our scheme is not dependent on the choice of wavelet, so a number of filter banks are used. The number of pyramidal levels is three $(1,2,3)$, At each successive level, number of directional sub bands are 8, 8, 4.Quantiation value is 64.Arnold transform time is 20.Matlab2008a, PhotoShop9.0 are used to test the robustness of the algorithm.

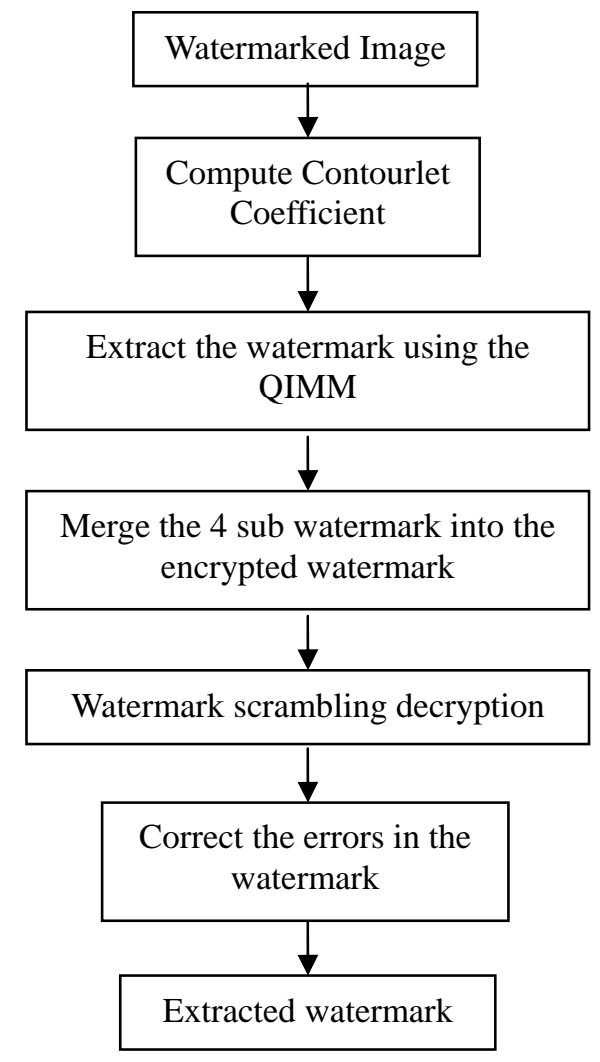

Figure 6.Steps of extraction

The error correction algorithm is implemented in dot net 2008. The given threshold is 3 pixel width and 3 pixel height. As a comparison, the watermarking scheme proposed in [13] is implemented whose related parameters are the same as that in authors' paper. Since the scheme integrate the watermarking error correction which is brand new, the uncorrected watermark is still provided .The error metrics used to test the proposed algorithm are peak signal to noise ratio (PSNR) and normalized cross correlation coefficient (NC).Let the host image of size $N_{1} \times N_{1}$ is $I_{m, n}$ and the watermarked counterpart is $\hat{I}_{m, n}$.PSNR in $\mathrm{dB}$ is given by

\section{Experimental Results}


$\operatorname{PSNR}=10 \lg \left(\frac{N_{1}{ }^{2} \max _{m, n} I_{m, n}^{2}}{\sum_{m, n}\left(I_{m, n}-\hat{I}_{m, n}\right)^{2}}\right)$

Let the watermark image be denoted by $w(i, j)$ and the extracted watermark be denoted by $w a(i, j)$ then NC is defined as

$$
N C=\frac{\sum_{i, j}[(w(i, j)-\bar{w}) \times(w a(i, j)-\overline{w a})]}{\sqrt{\sum_{i, j}(w(i, j)-\bar{w})^{2}} \times \sqrt{\sum_{i, j}(w a(i, j)-\overline{w a})^{2}}}
$$

Table 1 .Embedded Lena using different filter banks

\begin{tabular}{|l|l|}
\hline Transform(LP/D & Result/PSNR \\
FB) & \\
\hline 9-7/9-7 & \\
\hline Pkvaar & \\
\hline
\end{tabular}

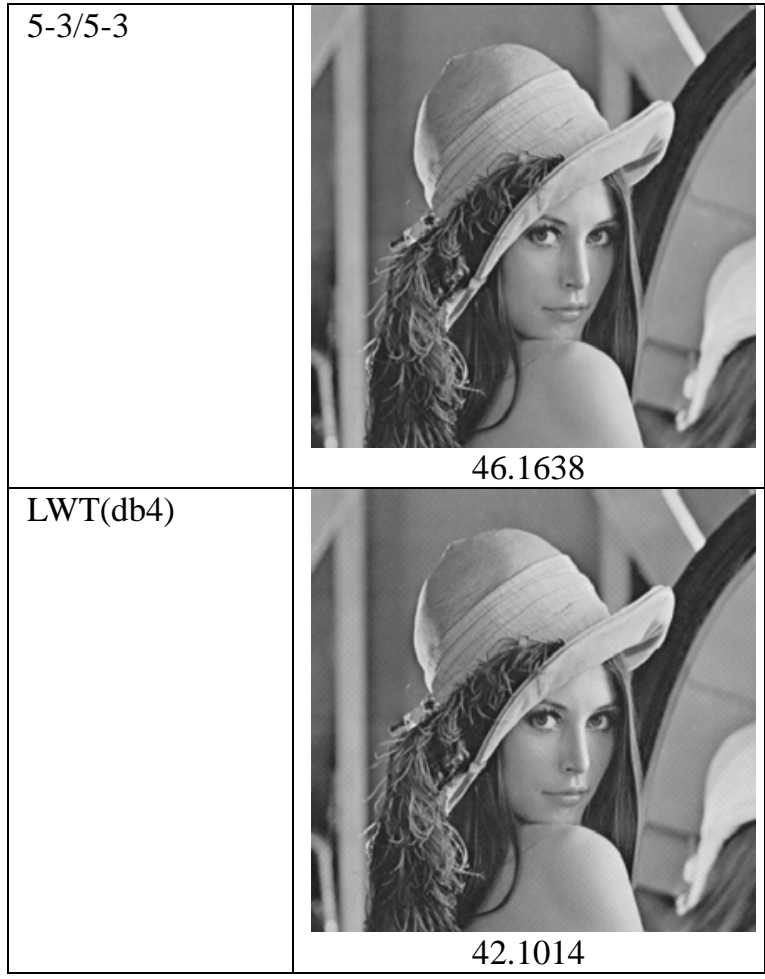

Table 2 .The extracted watermark using different filter bank under 3 common attacks (without error correction)

\begin{tabular}{|c|c|c|c|c|}
\hline LP/DFB & $\begin{array}{l}\text { Unattac } \\
\text { ked/NC }\end{array}$ & $\begin{array}{l}\text { JPEG( } \\
\mathrm{QF}=9 \text { ) } \\
\text { /NC }\end{array}$ & $\begin{array}{l}\text { Sharpe } \\
\text { ning/N } \\
\text { C }\end{array}$ & $\begin{array}{l}\text { Blurring } \\
\text { /NC }\end{array}$ \\
\hline haar/haar & $\begin{array}{c}W u \\
s t \\
0.9974\end{array}$ & $\begin{array}{l}w 4 \\
4 \\
0.6984\end{array}$ & $\begin{array}{l}4, \\
4, \\
0.4523\end{array}$ & $\begin{array}{l}\text { dx } \\
w^{2} \\
0.407\end{array}$ \\
\hline $9-7 / 9-7$ & $\begin{array}{l}w u \\
s \cdot t \\
0.9718\end{array}$ & $\begin{array}{l}w 4 \\
5 \\
0.7544\end{array}$ & $\begin{array}{c}44 \\
54 \\
0.6074\end{array}$ & $\begin{array}{c}\text { nu } \\
s^{\prime} \\
0.5216\end{array}$ \\
\hline pkva/pkva & $\begin{array}{l}W u \\
s t \\
0.9574\end{array}$ & $\begin{array}{l}w 4 \\
s t \\
0.7603\end{array}$ & $\begin{array}{l}4 y \\
s t \\
0.7167\end{array}$ & $\begin{array}{l}\text { wut } \\
5, y \\
0.5436\end{array}$ \\
\hline $5-3 / 5-3$ & $\begin{array}{l}44 \\
s, \\
0.7123\end{array}$ & $\begin{array}{l}\text { W1 } \\
\text { को } \\
0.4911\end{array}$ & $\begin{array}{c}{ }^{W} \\
x^{2} \\
0.3762\end{array}$ & 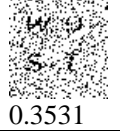 \\
\hline LWT(db4) & $\begin{array}{l}w_{\mathrm{u}} \\
\mathrm{s} \\
0.6611\end{array}$ & 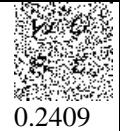 & 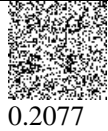 & 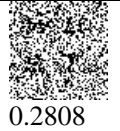 \\
\hline
\end{tabular}


Table 3 .The extracted watermark using different filter bank under 3 common attacks (with error correction)

\begin{tabular}{|c|c|c|c|c|}
\hline LP/DFB & $\begin{array}{l}\text { Unattack } \\
\text { ed/NC }\end{array}$ & $\begin{array}{l}\text { JPEG( } \\
\mathrm{QF}=9) \\
/ \mathrm{NC}\end{array}$ & $\begin{array}{l}\text { Sharpe } \\
\text { ning/N } \\
\text { C }\end{array}$ & $\begin{array}{l}\text { Blurri } \\
\text { ng/NC }\end{array}$ \\
\hline haar/haar & $\begin{array}{c}w u \\
s t \\
0.9736\end{array}$ & $\begin{array}{l}W \text { ts } \\
5 t \\
0.8918\end{array}$ & $\begin{array}{l}0 \\
0.5795 \\
0.5 \\
0.5\end{array}$ & 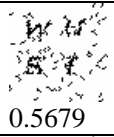 \\
\hline $9-7 / 9-7$ & $\begin{array}{c}w u \\
s t \\
0.9628\end{array}$ & $\begin{array}{c}W u \\
s t \\
0.9295\end{array}$ & $\begin{array}{l}\text { Whut } \\
51 . \\
0.8588\end{array}$ & $\begin{array}{c}W u \\
s, t \\
0.7824\end{array}$ \\
\hline pkva/pkva & $\begin{array}{c}W u \\
s t \\
0.9628\end{array}$ & $\begin{array}{c}W U \\
5 t \\
0.9400\end{array}$ & $\begin{array}{l}W u \\
s t \\
0.9315\end{array}$ & $\begin{array}{l}\text { We: } \\
5: \pm \\
0.7811\end{array}$ \\
\hline $5-3 / 5-3$ & $\begin{array}{c}\text { Wt w } \\
s t \\
0.9139\end{array}$ & 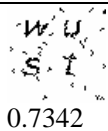 & 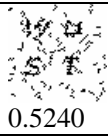 & 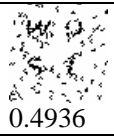 \\
\hline
\end{tabular}

Table 1 is the watermarked lena using different filter bank. The last row use the scheme proposed in [13] based on the lifting scheme wavelet transform whose filter name is ' $\mathrm{db} 4$ '.

Table 2 is the extracted watermark without the error correction step. The last row is using the lifting scheme wavelet transform (LWT). Table 3 is the extracted watermark with error correction.

\section{Discussion and Conclusion}

In this paper, an oblivious watermarking scheme based on contourlet transform and QIMM is presented. At the first stage, the watermark is scrambling encrypted and split into 4 equally sized sub images. In the second stage, watermark is embedded in the last LP level directional sub bands. Proposed method is robust to JPEG compression, sharpening and blurring. The major characteristics of proposed scheme can be summarized as follows:

(1) We applying the 3 level LP compositions, the watermark is embedded in the 4 directional sub bands in the last level which is different from the wavelet transform since one level wavelet transform has only 3 high pass images. Though the last level 4 directional sub band images belong to high pass image, we use a good quantization value to maintain both robustness and transparency. Though the scheme in [13] embedded 6 times original watermark, the scheme is prone to common signal processing proposed in this paper.
(2) Compared with the conventional watermarking scheme, in this paper watermark error correction is introduced to improve the quality of the extracted watermark which can be indicated by the above simulation. Conventional schemes can not effectively resolute the ownership authentication. If a scheme is robust to a kind of attack, seldom can this scheme be robust to another attack. With the help of properly designed error correction, watermarking schemes may have the ability to resist a number of attacks. We recommend that error correction should be involved in the new generation watermarking schemes.

(3) It's a fact in watermarking community that there is not a standard to judge the algorithm. If error correction is designed according to the characteristics of human visual system, NC can be viewed as a standard.

(4) We use different filter banks and achieved approximately the same results except haar filter bank. Though designing more effective directional filters of contourlet transform is an ongoing work [15], under the current contourlet transform the filter bank name can be used as secret key in watermark extraction process in practical situation.

However, this scheme is not robust to geometric attacks. The future work will focus on improving robustness against geometric attacks.

\section{References}

[1] Minh N. Do and M. Vitterli, "The Contourlet Transform: An efficient directional multiresolution image representation”, IEEE Transactions on Image Processing , pp.2091-2106,2005.

[2] Ho Seok Moon, Taewoo You, Myung Ho Sohn, Hye Soo Kim, Dong Sik Jang, "Expert system for low frequency adaptive image watermarking: Using psychological experiments on human image perception” , Expert Systems with Applications, pp. 674-686, 2007.

[3] Zhao Dawei, Chen Guanrong, Liu Wenbo, “A chaos-based robust wavelet-domain watermarking algorithm",Chaos,Solitons \& Fractals, pp . 47-54,2004.

[4] Mohammad Eyadat, Shantaram Vasikarla, "Performance evaluation of an incorporated DCT block-based watermarking algorithm with human visual system model” , Pattern Recognition Letters, pp. 1405-1411,2005.

[5] Xiaojun Qi, Ji Qi. "A robust content-based 
digital image watermarking scheme”, Signal Processing, Pages 1264-1280,2007.

[6] Xiang-yang Wang, Li-min Hou, Jun Wu, “A feature-based robust digital image watermarking against geometric attacks", Image and Vision Computing, pp. 980-989, 2008.

[7] B. Chen and G.W. Wornell, "Quantization index modulation: A class of provably good methods for digital watermarking and information embedding”, IEEE Transaction. Information Theory, pp. 1423- 1443,2001.

[8] M. Jayalakshmi, S.N. Merchant, and U.B. Desai, "Digital Watermarking in Contourlet Domain", Pattern Recognition, 2006. ICPR 2006. 18th International Conference,pp.861 - 864, 2006

[9] B. Chandra Mohan and S. Srinivas Kumar, "Robust Digital Watermarking Scheme using Contourlet Transform”, IJCSNS International Journal of Computer Science and Network Security, pp.43-51,2008.

[10] M. Jayalakshmi, S. N. Merchant, U. B. Desai, "Significant Pixel Watermarking in Contourlet Domain” , IET International Conference on Visual Information Engineering(VIE2006), pp. 416- 421,2006.

[11] Ali Bouzidi, Nadia Baaziz, "Contourlet Domain Feature Extraction for Image Content Authentication”, Multimedia Signal Processing, 2006 IEEE 8th Workshop,pp.202-206, 2006.

[12] Haohao Song, Songyu Yu, Xiaokang Yang, Li Song, Chen Wang, "Contourlet-based image adaptive watermarking”, Signal Processing: Image Communication, pp.162-178, 2008.

[13] Dongfang Chen, Youqing Zhang, "A Blind watermarking algorithm Based on Lifting Scheme Wavelet and encrypted watermark" , Computer Applications,pp.615-619, 2008.

[14] M. Ramkumar and A. N. Akansu, "A Robust Oblivious Watermarking Scheme”, IEEE International Conference on Image Processing (ICIP'2000), Vancouver, Canada, pp.450-453, 2000.

[15] Po Duncan D, Do M N. "Directional Multiscale modeling of images using the contourlet transform” , IEEE Transactions on Image Processing, pp.1610-1620,2006.

[16] I.J.Cox,U. Kilian,F.T.Leighton, "Secure Spread Spectrum Watermarking for Multimedia”.IEEE Transactions on Image Processing, pp. 1673-1687, 1997. 